\title{
A Three-Dimensional Cooperative Guidance Law of Multimissile System
}

\author{
Xing Wei, Yongji Wang, Shuai Dong, and Lei Liu \\ Key Laboratory of Ministry of Education for Image Processing and Intelligent Control, School of Automation, \\ Huazhong University of Science and Technology (HUST), Wuhan 430074, China
}

Correspondence should be addressed to Yongji Wang; wangyjch@mail.hust.edu.cn

Received 15 May 2015; Accepted 13 September 2015

Academic Editor: Christopher J. Damaren

Copyright (C) 2015 Xing Wei et al. This is an open access article distributed under the Creative Commons Attribution License, which permits unrestricted use, distribution, and reproduction in any medium, provided the original work is properly cited.

In order to conduct saturation attacks on a static target, the cooperative guidance problem of multimissile system is researched. A three-dimensional guidance model is built using vector calculation and the classic proportional navigation guidance (PNG) law is extended to three dimensions. Based on this guidance law, a distributed cooperative guidance strategy is proposed and a consensus protocol is designed to coordinate the time-to-go commands of all missiles. Then an expert system, which contains two extreme learning machines (ELM), is developed to regulate the local proportional coefficient of each missile according to the command. All missiles can arrive at the target simultaneously under the assumption that the multimissile network is connected. A simulation scenario is given to demonstrate the validity of the proposed method.

\section{Introduction}

Saturation attack, which involves simultaneous attack from different missiles in a communication network, is an important combat manner to penetrate the missile defence system. In fact, a group of well-organized missiles of low cost and poor performance may yield better results than a single excellent one. The key to cooperative guidance of multimissile system is that all missiles reach the target at the same time in the case of saturation attacking.

Cooperative control theories have been researched broadly with respect to different agents, such as unmanned aerial vehicle [1-3], satellite [4], and some abstract objects [5, 6]. A cooperative control strategy for achieving cooperative timing among teams of vehicles which is based on coordination variables and functions was developed [7]. However, there are only a few existing literatures considering the cooperative timing problem of missiles. In [8], with a combination of the PNG law and the feedback of the impact time error, an impact time control guidance (ITCG) law for salvo attack of antiship missiles was presented and could be used to guide multiple missiles to hit a stationary target simultaneously at a desirable impact time. Based on this law, a cooperative PNG law was proposed by introducing a new concept of the variance of time-to-go of multiple missiles in [9]. With the weighting average consensus algorithm [10], a cooperative guidance scheme [11] was developed based on the ITCG law.

Different from the unmanned aerial vehicle or satellite, the maneuver flight of missile is mainly based on aerodynamic force and its guidance does not include task assignment, cooperative path planning, and path tracking. This makes it particularity hard to design a cooperative strategy for the multimissile system. In the case of a group of missiles intercepting a single maneuver target, an optimal cooperative guidance law [12] based on comprehensive cost function of missiles was derived with the constraint of a relative intercept angle. A time-cooperative guidance architecture which is a centralized coordination control form was proposed based on leader-follower strategy [13]. Using the dynamic surface control theory and disturbance observation technology, [14] developed a novel integrated guidance and control law, which can realize cooperation of impact time and flight position for multiple missiles during their cooperative attack. All these methods adopt a centralized control manner in which one missile must exchange information with all the other missiles.

Many classical literatures [15-18] described and analysed the PNG law in two-dimensional plane, and most of the three-dimensional trajectory simulations [19] generally 
adopted the dimensionality reduction method to decompose the movement of missile into longitudinal and lateral plane. Then PNG law was utilized to obtain the trajectory in two planes, respectively, and thereby compounded the desired three-dimensional trajectory. However, this method needs to solve some antitrigonometric functions [20] on account of the direction of coordinate axis; meanwhile the coupled problems have adverse effects on the precision. Furthermore, geometric methods mentioned above are on a necessary condition that the velocities of all missiles are constant. In order to settle these problems, this paper introduces the space vector method to generate the PNG law in three dimensions.

In order to achieve the simultaneous attack of missiles, the consensus of time-to-go (remaining flight time, i.e., arriving time) is considered in this paper. From the previous literature, it is known that the time-to-go of missiles can be changed by adjusting proportional coefficient $k$ of the PNG law. However, the relationship between the $t_{\text {go }}$ command and the local proportional guidance law is nonlinear and hard to obtain directly, especially in three dimensions, and then the fitting methods are considered. An artificial neural network (ANN), with a fixed number of numeric inputs and outputs, can be regarded as a complex nonlinear function. By learning iteratively large numbers of samples, the ANN stores the mapping relation between inputs and outputs. Due to this ability of self-learning and self-adaption, ANN is widely used in expert systems. Although ANN is of high precision, the learning speed of traditional ANN is so slow that it cannot be used in online cooperative guidance. ANN with extreme learning machine (ELM) is introduced in the paper to overcome this problem. It is notable that ELM is of strong generalization ability [21], which can learn the rules more accurately from training data; meanwhile it can obtain the appropriate output even if the input data is out of training set. Therefore, the problem of the inaccurate results caused by some special inputs of ANN is solved.

In this paper, we design a cooperative guidance strategy to achieve simultaneous attack based on expert system using ELM, which just requires that the communication network is connected. Considering that the communication between missiles might be incomplete on account of the disturbances from defense system and other environment factors, the centralized cooperative guidance strategy is liable to fail and the distributed strategy is more effective. Via a distributed protocol through the connected network, which aims at asymptotical consensus of time-to-go commands, the $t_{\mathrm{go}}$ commands of all missiles are coordinated. Then the local proportional guidance law of each missile is regulated by the expert system according to the $t_{\mathrm{go}}$ commands. Through the consensus algorithm of distributed cooperative guidance strategy, the proportional coefficients of all missiles are adjusted to achieve saturation attack.

The remainder of this paper is organized as follows. Section 2 describes the cooperative guidance problem of multiple missiles on three dimensions. Section 3 proposes a new distributed cooperative guidance strategy based on the expert system with ANNs using ELM. Section 4 shows the simulation results to illustrate the validity of the proposed

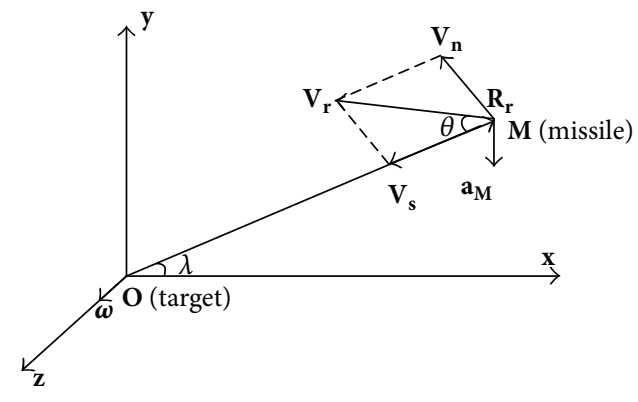

FIGURE 1: The relative movement of target and missile.

algorithm. Finally, some concluding remarks to this paper are presented in Section 5.

\section{Cooperative Guidance Problem Formulation}

Consider the cooperative guidance of $n$ similar missiles which are denoted by $1,2, \ldots, n$. The objective is to make all missiles arrive at a static target at the same time. First, the coordinate system which is used to describe the relative movement of missile and target in space is built, and the three-dimensional guidance model is obtained. Then, the PNG law is extended to three dimensions. At last, the cooperative guidance problem of multiple missiles is described.

2.1. Movement Coordinate System. Set $\mathbf{V}_{\mathbf{r}}$ as the relative velocity vector of missile to the target and $\mathbf{R}_{\mathbf{r}}$ as the relative position vector (sight vector). Assume that the plane composed of $\mathbf{V}_{\mathbf{r}}$ and $\mathbf{R}_{\mathbf{r}}$ is instantaneous sight transfer plane at any time. $\mathbf{V}_{\mathbf{s}}$ and $\mathbf{V}_{\mathbf{n}}$ are the projection elements of $\mathbf{V}_{\mathbf{r}}$ on direction and vertical direction of $\mathbf{R}_{\mathbf{r}}$, respectively. The relative movement coordinate system is depicted in Figure 1. The origin $\mathbf{O}$ is located on the target. The $\mathbf{x O y}$ plane is instantaneous sight transfer plane and axis $\mathbf{x}$ is on the horizontal plane of target which points to the missile. Axis $y$ is on the plumb line of target which points up and axis $\mathbf{z}$ is perpendicular to the $\mathbf{x O y}$ plane. $\lambda$ is the line of sight (LOS) angle and $\theta$ is the included angle of $\mathbf{V}_{\mathbf{r}}$ and $\mathbf{R}_{\mathbf{r}}$. $\boldsymbol{\omega}$ is the LOS angular velocity vector and $\mathbf{a}_{\mathbf{M}}$ is the command acceleration vector of missiles.

Let $\mathbf{R}_{\mathbf{M}}$ and $\mathbf{R}_{\mathbf{T}}$ denote the position vector of missiles and target and $\mathbf{V}_{\mathbf{M}}$ and $\mathbf{V}_{\mathrm{T}}$ denote the velocity vector, respectively. Then, we obtain relative position vector $\mathbf{R}_{\mathbf{r}}=\mathbf{R}_{\mathrm{M}}-\mathbf{R}_{\mathrm{T}}$ and relative velocity vector $\mathbf{V}_{\mathbf{r}}=\mathbf{V}_{\mathbf{M}}-\mathbf{V}_{\mathrm{T}}$. Assume that the target is static in the following design of cooperative guidance law; that is, $\mathbf{R}_{\mathbf{T}}=\mathbf{V}_{\mathrm{T}}=\mathbf{0}$. It only needs to consider the motion of missile as follows:

$$
\begin{aligned}
& \frac{\mathrm{d} \mathbf{V}_{\mathbf{M}}}{\mathrm{d} t}=\mathbf{a}_{\mathbf{M}}, \\
& \frac{\mathrm{d} \mathbf{R}_{\mathbf{M}}}{\mathrm{d} t}=\mathbf{V}_{\mathbf{M}} .
\end{aligned}
$$

2.2. Three-Dimensional PNG Law. In the relative movement coordinate system, the command acceleration vector $\mathbf{a}_{M}$ of $\mathrm{PN}$ is perpendicular to LOS, and the magnitude is 
proportional to the approach velocity and the LOS angular rate; that is,

$$
\left|\mathbf{a}_{\mathbf{M}}\right|=k \cdot\left|\mathbf{V}_{\mathbf{s}}\right| \cdot|\boldsymbol{\omega}|
$$

where $k$ denotes the proportional coefficient. In general, large $k$ results in sensitivity to noises, while small $k$ leads to slow the response of the navigation against a quick target. Besides, the overload imposed on the missile is limited; the choice of $k$ is usually $2 \leq k \leq 6$ [22].

From the three-dimensional model, it is easy to know that the LOS angular velocity vector $\boldsymbol{\omega}$ is perpendicular to the sight transfer plane $\mathbf{x O y}$ and along the $\mathbf{z}$ direction. Analyzing the two components of $\mathbf{V}_{\mathbf{r}}$, it can be found that the rotation of LOS is caused by $\mathbf{V}_{\mathbf{n}}$, while $\mathbf{V}_{\mathbf{s}}$ only makes the missile approach the target. Therefore, the magnitude of $\boldsymbol{\omega}$ has the direct relationship with $\mathbf{V}_{\mathbf{n}}$. The two input parameters of the guidance law are both determined by relative velocity vector $\mathbf{V}_{\mathbf{r}}$, so the problem is transformed into the decomposition of $\mathbf{V}_{\mathbf{r}}$. It can be represented by the dot product and cross product of the relative velocity vector and position vector:

$$
\begin{aligned}
& \left|\mathbf{V}_{\mathbf{s}}\right|=\left|\mathbf{V}_{\mathbf{r}}\right| \cdot|\cos \theta|=\frac{\left|\mathbf{V}_{\mathbf{r}} \cdot \mathbf{R}_{\mathbf{r}}\right|}{\left|\mathbf{R}_{\mathbf{r}}\right|}, \\
& \left|\mathbf{V}_{\mathbf{n}}\right|=\left|\mathbf{V}_{\mathbf{r}}\right| \cdot|\sin \theta|=\frac{\left|\mathbf{V}_{\mathbf{r}} \times \mathbf{R}_{\mathbf{r}}\right|}{\mathbf{R}_{\mathbf{r}}} .
\end{aligned}
$$

Suppose that the mass of the missile is $m$, and the momentum is $\mathbf{P}=m \mathbf{V}_{\mathbf{r}}$. So the angular momentum $\mathbf{L}$ to the origin is described by velocity as

$$
\mathbf{L}=\mathbf{R}_{\mathbf{r}} \times \mathbf{P}=m \mathbf{R}_{\mathbf{r}} \times \mathbf{V}_{\mathbf{r}} .
$$

Additionally, the moment of inertia to the origin can be expressed as $I=m \mathbf{R}_{\mathbf{r}}^{2}$, and angular momentum $\mathbf{L}$ is represented by angular velocity as

$$
\mathbf{L}=I \boldsymbol{\omega}=m \mathbf{R}_{\mathbf{r}}^{2} \boldsymbol{\omega} .
$$

From expression (4) and (5), we can obtain the angular velocity vector $\boldsymbol{\omega}$ as follows:

$$
\omega=\frac{\mathbf{R}_{\mathbf{r}} \times \mathbf{V}_{\mathbf{r}}}{\mathbf{R}_{\mathbf{r}}^{2}} .
$$

Since the magnitude of $\mathbf{a}_{\mathbf{M}}$ is obtained, then it is needed to discuss its direction. The aim of command acceleration is to eliminate the LOS angular speed and make the relative velocity along the LOS direction. Moreover, from the above analysis, it is known that the LOS angular rate is caused by $\mathbf{V}_{\mathbf{n}}$. Thus, the command acceleration can be used to produce a velocity of opposite direction to $\mathbf{V}_{\mathbf{n}}$, in order to make the relative speed of this direction equal to zero. The best choice of $\mathbf{a}_{\mathbf{M}}$ is parallel to $\mathbf{V}_{\mathbf{n}}$ and in the opposite direction. It is noted that $\boldsymbol{\omega}$ is perpendicular to the plane $\mathbf{x O y}$ and $\mathbf{a}_{M}$ is perpendicular to LOS. So, the direction of $\mathbf{a}_{\mathbf{M}}$ is the same as the cross product of $\boldsymbol{\omega}$ and $\mathbf{V}_{\mathbf{s}}$. Additionally, $\boldsymbol{\omega}$ is perpendicular to $\mathbf{V}_{\mathbf{s}}$, and then it has

$$
\left|\omega \times V_{s}\right|=|\omega| \cdot\left|V_{s}\right| \text {. }
$$

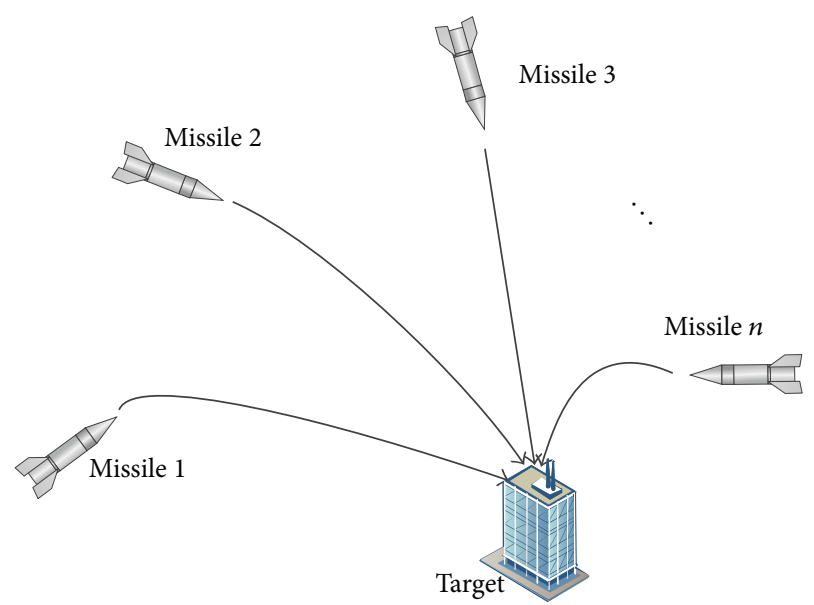

FIGURE 2: The concept map of multimissiles cooperative saturation attack.

Compared to expression (2), the PNG law can be extended to the three dimensions as follows:

$$
\mathbf{a}_{\mathbf{M}}=k \boldsymbol{\omega} \times \mathbf{V}_{\mathbf{s}} .
$$

On account of the fact that the direction of $\mathbf{V}_{\mathbf{s}}$ is opposite to relative position vector $\mathbf{R}_{\mathbf{r}}$, combined with expression (3), vector $\mathbf{V}_{\mathbf{s}}$ can be obtained as follows:

$$
\mathbf{V}_{\mathbf{s}}=-\frac{\left|\mathbf{V}_{\mathbf{s}}\right|}{\left|\mathbf{R}_{\mathbf{r}}\right|} \mathbf{R}_{\mathbf{r}}=-\frac{\left|\mathbf{V}_{\mathbf{r}} \cdot \mathbf{R}_{\mathbf{r}}\right|}{\left|\mathbf{R}_{\mathbf{r}}\right|^{2}} \mathbf{R}_{\mathbf{r}} .
$$

Considering that the cooperative guidance problem is to make a series of missiles attack the target simultaneously, the distance between missile and target is decreasing and the symbol of $\mathbf{V}_{\mathbf{r}} \cdot \mathbf{R}_{\mathbf{r}}$ is negative all the time. Thus, the sign of absolute value can be removed.

Substitute (6) and (9) into guidance law (8); it has

$$
\mathbf{a}_{\mathbf{M}}=k \frac{\mathbf{R}_{\mathbf{r}} \cdot \mathbf{V}_{\mathbf{r}}}{\left|\mathbf{R}_{\mathbf{r}}\right|^{4}}\left(\mathbf{R}_{\mathbf{r}} \times \mathbf{V}_{\mathbf{r}}\right) \times \mathbf{R}_{\mathbf{r}} .
$$

Next, the cooperative guidance problem of multiple missiles is introduced. Almost all of the variables including position and velocity vectors are time-variant and the subscript $i$ of a variable denotes that it belongs to the $i$ th missile for convenience.

2.3. Cooperative Guidance Problem of Multiple Missiles. How multimissiles cooperatively attack the ground static target is shown in Figure 2. $n$ missiles fly towards the target from different distances and directions, respectively; besides, their communication topology is undirected and strongly connected. Namely, every missile can only exchange information with its neighbors.

Then the objective of cooperative guidance is to find the appropriate acceleration command $\mathbf{a}_{\mathbf{M}}$ and make all missiles reach the target simultaneously; that is, $r_{1}\left(t_{f}\right)=r_{2}\left(t_{f}\right)=$ $\cdots=0$. The time-to-go of missile $i$ is a monotonic decreasing function of $k_{i}$, so we can regulate $k_{i}$ to adjust the arrival time. 


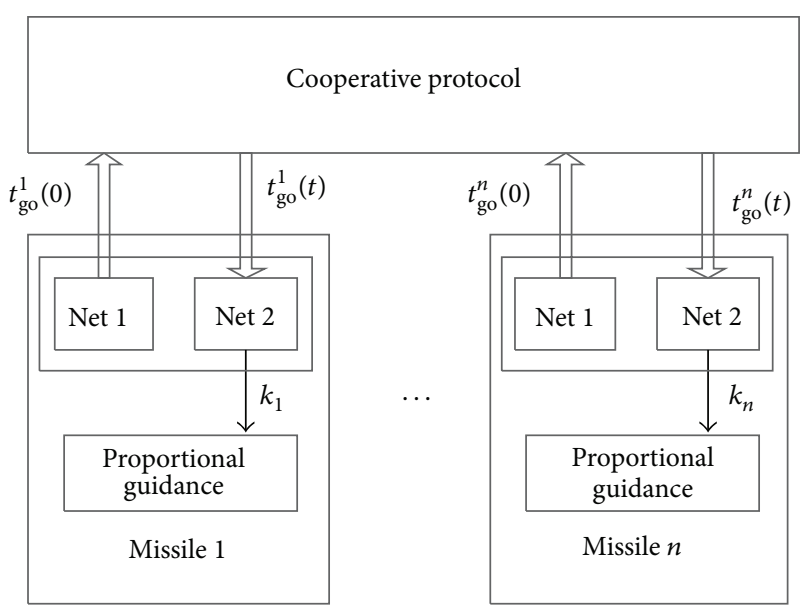

FIGURE 3: The framework of cooperative guidance strategy.

In order to achieve simultaneous attack, a distributed cooperative guidance strategy is adopted as shown in Figure 3. The protocol makes the expected $t_{\mathrm{go}}$ of all missiles achieve consensus asymptotically. Then, consisting of ANNs trained offline, these local expert systems transform the $t_{\mathrm{go}}$ command to corresponding proportional coefficient $k$. The details will be introduced in the next section.

\section{Algorithm Description}

Denote $t_{\mathrm{go}}$ of missile $i$ by $T_{i}$ for convenience. Since the closed loop system of (1) is autonomous, if $\mathbf{R}_{\mathbf{M i}}\left(t_{0}\right)$ and $\mathbf{V}_{\mathbf{M i}}\left(t_{0}\right)$ are known and $k_{i}(t)=k_{i}\left(t_{0}\right)$ when $t \geq t_{0}$, then $T_{i}\left(t_{0}\right)$ is determined by the function

$$
T_{i}\left(t_{0}\right)=f\left(\mathbf{R}_{\mathbf{M i}}\left(t_{0}\right), \mathbf{V}_{\mathbf{M i}}\left(t_{0}\right), k_{i}\left(t_{0}\right)\right) .
$$

Similarly, when the required time-to-go $T_{i}\left(t_{0}\right)$ is given, there exists corresponding $k_{i}\left(t_{0}\right)$ that can be obtained by

$$
k_{i}\left(t_{0}\right)=f^{-1}\left(\mathbf{R}_{\mathbf{M i}}\left(t_{0}\right), \mathbf{V}_{\mathbf{M i}}\left(t_{0}\right), T_{i}\left(t_{0}\right)\right) .
$$

Figure 4 shows some curves of $T_{i}\left(t_{0}\right)$ with respect to $k_{i}\left(t_{0}\right)$. Although it is hard to derive the analytic form of the function $f(\cdot)$ and its inverse $f^{-1}(\cdot)$, fortunately it can resort to the ANNs.

3.1. Extreme Learning Machine. ELM is a simple learning algorithm for single-hidden layer feedforward neural networks (SLFNs) which achieves fast learning through increasing the number of hidden nodes and obtains good generalization performance [21]. In the training process with ELM, the input weights (linking the input layer to the hidden layer) and hidden layer biases of SLFNs can be assigned arbitrarily and do not need to adjust. After being chosen randomly, SLFNs can be simply considered as a linear system and the output weights (linking the hidden layer to the output layer) can be analytically determined through simple generalized inverse operation of the hidden layer output matrices. Only by setting the number of hidden nodes is it easy to get the single optimal

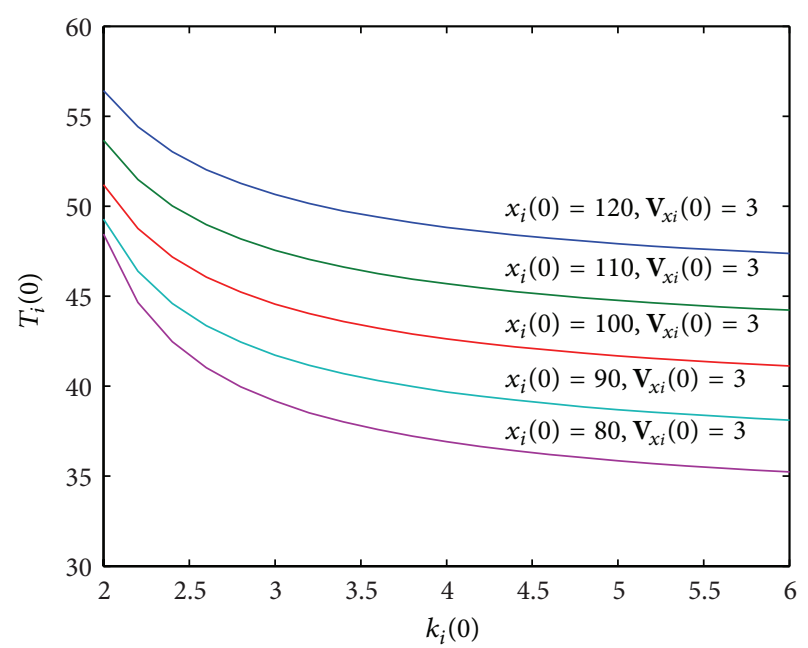

FIGURE 4: The mapping relation between proportional guidance coefficient and time-to-go.

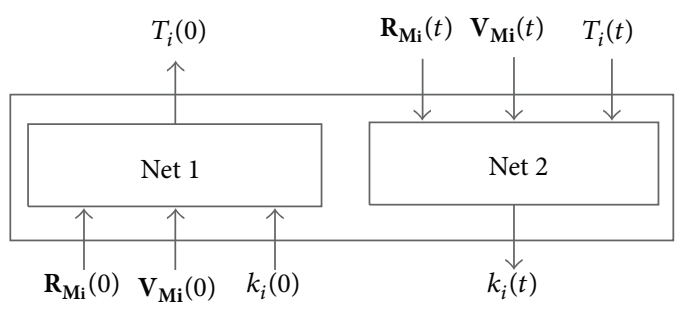

FIGURE 5: Expert system with two SLFNs.

solution of SLFNs with ELM which has a learning speed much faster than traditional feedforward neural network learning algorithms like back-propagation (BP) algorithm while obtaining better generalization performance. ELM not only tends to reach the smallest training error but also obtains the smallest norm of weights.

Compared with BP, ELM only needs to adjust the number of hidden nodes and avoids the multiple iterations; hence, it avoids the problem of local minimum or infinite training iteration and can reach the minimum training error. Owing to the strong generalization ability of ELM, the precision of training results can be guaranteed as long as the range of sample data is appropriate.

3.2. Distributed Cooperative Guidance Strategy. In order to approximate $f(\cdot)$ and $f^{-1}(\cdot)$, we build two SLFNs and train them with plentiful simulation data beforehand under ELM. Then introduce $T_{i}$ as coordination variables; the structure of cooperative guidance strategy is built as shown in Figure 3. These two SLFNs get assembled into an expert system as shown in Figure 5. Net 1 is the approximation of $f(\cdot)$ and used to evaluate the $T_{i}(0)$ as the initial state under the state of missile at the beginning of cooperative guidance; Net 2 is the approximation of $f^{-1}(\cdot)$ and used to calculate $k_{i}(t)$ according to $T_{i}(t)$ in real time.

The time-variant undirected graph [23] of the multimissile system is defined as $G(t)=\{V, E(t)\}$, where $V=$ 
$\{1,2, \ldots, n\}$ is the set of all missiles and $E(t)=\{(i, j) \in V \times V$ : $i \sim j\}$ is a set of edges, in which the edge $i \sim j$ means that missiles $i$ and $j$ can exchange with each other. The Laplacian matrix $L(t)$ is defined as

$$
\begin{aligned}
& L(t)=D(t)-A(t)=D(t)-\left(a_{i j}(t)\right)_{n \times n}, \\
& a_{i j}(t)= \begin{cases}1, & \text { if }(i, j) \in E(t) \\
0, & \text { otherwise, }\end{cases} \\
& D(t)=\operatorname{diag}\left\{\sum_{j=1}^{n} a_{1 j}(t), \sum_{j=1}^{n} a_{2 j}(t), \ldots, \sum_{j=1}^{n} a_{n j}(t)\right\},
\end{aligned}
$$

where $D(t)$ is the degree matrix and $A(t)$ is the adjacency matrix of the graph. If $G(t)$ is connected, there exists $L(t)=$ $L^{T}(t) \geq 0$

If there is no cooperation among missiles, the original $T_{i}(t)$ satisfies

$$
\begin{aligned}
& \dot{T}_{i}(t)=-1, \\
& T_{i}(t)=T_{i}(0)-t .
\end{aligned}
$$

In order to make $T_{i}(t), i=1,2, \ldots, n$, achieve consensus, we choose the distributed cooperative protocol below for missile $i$ :

$$
\dot{T}_{i}(t)=-1-c_{i} \sum_{j \in N_{i}}\left[T_{i}(t)-T_{j}(t)\right]
$$

where $N_{i}$ is the neighborhood (the set of missiles that can exchange information with $i$ ) of missile $i$ and $c_{i}$ is specified as

$$
\begin{aligned}
& c_{i}>0, \quad \text { if } \sum_{j \in N_{i}}\left[T_{i}(t)-T_{j}(t)\right]<0, \\
& c_{i}=0, \quad \text { otherwise. }
\end{aligned}
$$
that

The purpose of $c_{i}$ is that all $T_{i}$ converge to $\max _{i}\left\{T_{i}\right\}$ and

$$
T_{i}(t) \geq T_{i}(0)-t,
$$

which means $k_{i}(t) \leq k_{i}(0)$

From Figure 4, we can see that $T_{i}$ decreases with $k_{i}$ increasing. Through decreasing $k$ of the missile with less time-to-go, the practical flight time of it can be added. The range of traditional proportional guidance coefficient is $[2,6]$. If $k_{i}(0)$ is specified as 6 , then $k_{i}(t) \leq 6$.

Theorem 1. System (15) will achieve consensus asymptotically.

Proof. Let $T=\left[\begin{array}{llll}T_{1} & T_{2} & \cdots & T_{n}\end{array}\right]^{T}$ and $C=\operatorname{diag}\left\{c_{1}, c_{2}, \ldots, c_{n}\right\}$; then (15) can be rewritten as

$$
\dot{T}=-\mathbf{1}_{n \times 1}-C L T .
$$

Define energy function

$$
Q(T)=\frac{1}{4} \sum_{i=1}^{n} \sum_{j \in N_{i}}\left(T_{i}-T_{j}\right)^{2} ;
$$

then, the derivate is

$$
\dot{Q}=\frac{1}{2} \sum_{i=1}^{n} \sum_{j \in N_{i}}\left(T_{i}-T_{j}\right)\left(\dot{T}_{i}-\dot{T}_{j}\right)=T^{T} L \dot{T}
$$

By substituting (18) into (20), we can obtain

$$
\dot{Q}=-T^{T} L \mathbf{1}_{n \times 1}-T^{T} L C L T .
$$

Because $\mathbf{1}_{n \times 1}$ can be regarded as the eigenvector of matrix $L$ under the eigenvalue of zero, $L \mathbf{1}_{n \times 1}=\mathbf{0}$. Besides, $L C L \geq 0$; then we have

$$
\dot{Q}=-T^{T} L C L T \leq 0,
$$

where the condition of $L C L=0$ is $T_{1}=T_{2}=\cdots=T_{n}$; hence,

$$
\lim _{t \rightarrow \infty}\left(T_{i}-T_{j}\right)=0, \quad \forall i, j \in V
$$

which means that system (15) will achieve consensus asymptotically.

Usually, we should choose big $c_{i}$ to accelerate the convergence rate of system (15). Furthermore, when $\max \left\{\left|T_{i}-T_{j}\right|\right\}$ decreases into the allowable tolerance scope at $t^{*}$, we can stop cooperative guidance and fix $k_{i}=k_{i}\left(t^{*}\right)$. The reason is that a longer convergence process may require a wider varying range of $r_{i}\left(t_{0}\right)$ and $\lambda_{i}\left(t_{0}\right)-\theta_{i}\left(t_{0}\right)$ of the samples for these two SLFNs. This will lead to longer training time and more complicated topological structure.

\section{Simulation and Results}

In this section, a scenario is given to illustrate the proposed cooperative guidance strategy [9]. Consider the saturation attack of 5 missiles on a static target. The initial states of these missiles and target are listed as

$$
\begin{aligned}
R_{M 1} & =(90,12,6) \mathrm{m}, \\
V_{M 1} & =(-4.2,0.8,0.6) \mathrm{m} / \mathrm{s}, \\
R_{M 2} & =(95,11,5) \mathrm{m}, \\
V_{M 2} & =(-3.7,0.9,0.5) \mathrm{m} / \mathrm{s}, \\
R_{M 3} & =(98,10,3) \mathrm{m}, \\
V_{M 3} & =(-3.5,0.8,0.8) \mathrm{m} / \mathrm{s}, \\
R_{M 4} & =(93,9,4) \mathrm{m}, \\
V_{M 4} & =(-4.0,0.7,0.6) \mathrm{m} / \mathrm{s}, \\
R_{M 5} & =(94,8,6) \mathrm{m}, \\
V_{M 5} & =(-3.8,0.9,0.7) \mathrm{m} / \mathrm{s}, \\
R_{T} & =(0,0,0) \mathrm{m}, \\
V_{T} & =(0,0,0) \mathrm{m} / \mathrm{s} .
\end{aligned}
$$




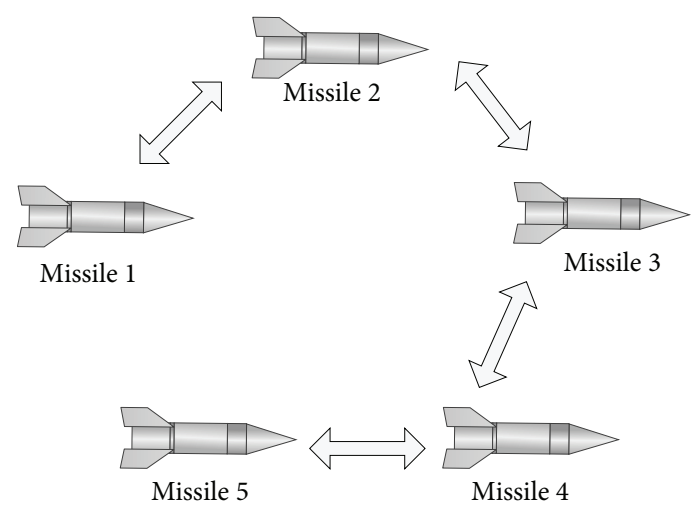

FIGURE 6: Topological structure of the network.

The topological structure of the communication network among them is shown in Figure 6 and the corresponding Laplacian matrix is

$$
L=\left[\begin{array}{ccccc}
1 & -1 & 0 & 0 & 0 \\
-1 & 2 & -1 & 0 & 0 \\
0 & -1 & 2 & -1 & 0 \\
0 & 0 & -1 & 2 & -1 \\
0 & 0 & 0 & -1 & 1
\end{array}\right]
$$

With the algorithm of ELM and Neural Network Toolbox of Matlab, these two SLFNs can be built and trained conveniently. Each SLFN includes 250 neurons in the hidden layer and adopts activation functions "tansig" and "purelin" for the hidden layer and output layer, respectively. In order to generate enough samples, the guidance of a single missile is simulated with the fixed proportional guidance coefficient iteratively, with the initial conditions $R_{M}(0), V_{M}(0)$, and $k(0)$ varying at points

$$
\begin{gathered}
x_{M}(0)=120:-10: 70, \\
y_{M}(0)=20:-5: 0, \\
z_{M}(0)=10:-5: 0, \\
V_{M x}(0)=-5.0: 1.0: 3.0, \\
V_{M y}(0)=1.0:-0.5: 0, \\
V_{M z}(0)=1.0:-0.5: 0, \\
k(0)=2.1: 0.1: 6 .
\end{gathered}
$$

The training results of the SLFN with 250 hidden neurons using ELM are quite good. The error of mean square is 0.0069 , which is close to the result of BP. However, the training time is only 4.3 seconds, which is much less than BP of 51.1 seconds. When increasing the number of hidden neurons to 500 , the training time with ELM still needs only 9.5 seconds but the training results improve significantly. In conclusion, the training speed and fitting precision of ELM both have a great advantage over other ANN algorithms.

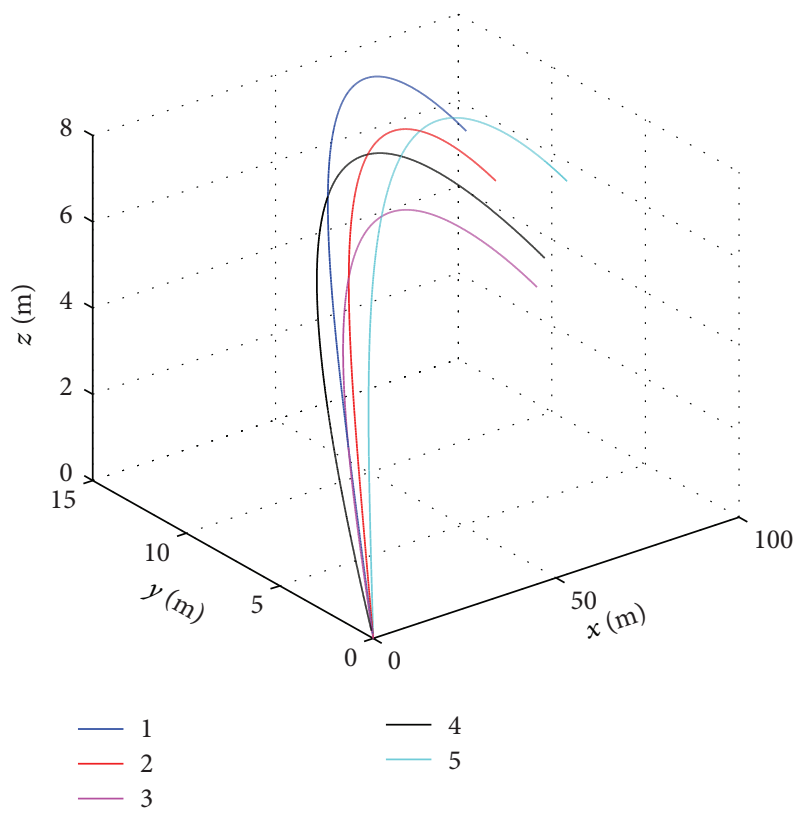

FIgURE 7: The trajectories of missiles.

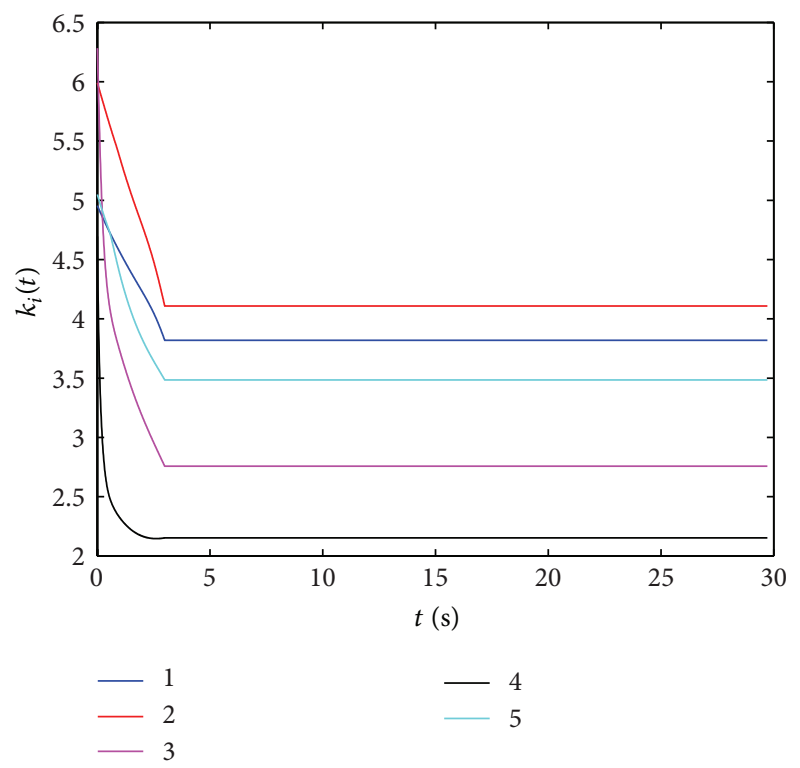

FIGURE 8: The trajectories of proportional coefficients.

With the two trained SLFNs, setting the parameters $c_{i}=5$ and proportional coefficients $k_{i}(0)=6$, the simulation is conducted and results are depicted in Figures 7-9. When $t=3 \mathrm{~s}$, the time-to-go commands have already converged to $T_{3}(t)$. So we stop the cooperative guidance and fix the proportional guidance coefficients. The result is that all missiles arrive at the target almost at the same time $t=29.72 \mathrm{~s}$. It is worth noting that $k_{i}(t) \leq 6$ always. Compared with results of [9], it is obvious that the convergence rate of time-to-go is much faster, and the variation range of proportional coefficients is smaller (not more than 6), without achieving the same end value. In all, the distributed cooperative guidance strategy provides better flexibility and autonomy for each missile. 


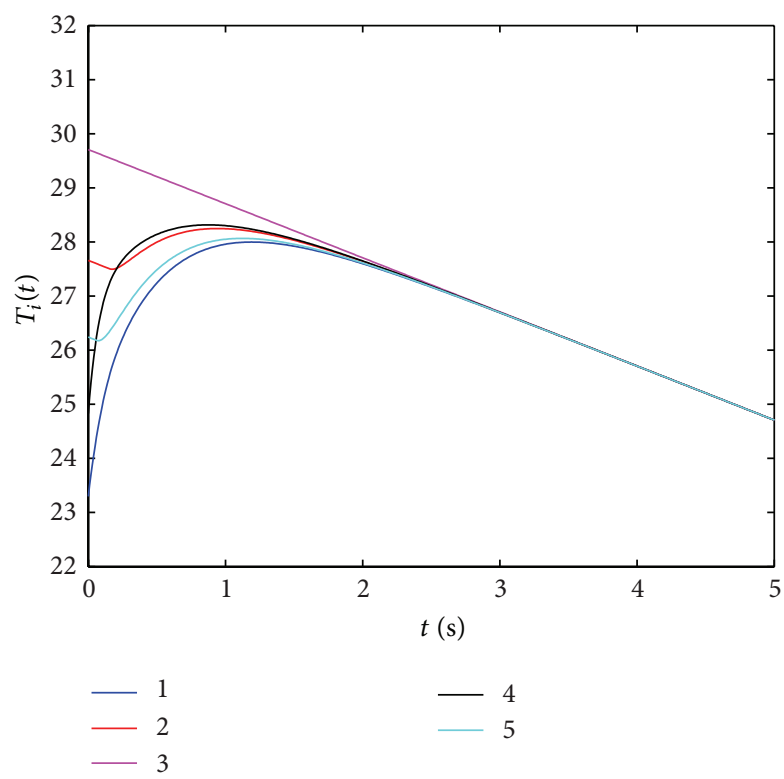

FIGURE 9: The converging process of time-to-go.

\section{Conclusion}

In this paper, the three-dimensional cooperative guidance for simultaneous attack on a static target of multimissile system is investigated. The classic PNG law is extended to three dimensions using vector operation; besides, an indirect and distributed cooperative proportional guidance strategy, based on expert systems and first-order $t_{\text {go }}$ consensus protocol, is proposed. This strategy can achieve simultaneous attack by adjusting $t_{\text {go }}$ commands cooperatively and guarantee that the overload satisfies limitation of the missile body since the proportional guidance coefficient is always less than 6 in the simulation. Simulation results demonstrate the performance of this proposed cooperative guidance strategy. Future studies will be conducted on the cooperative guidance of simultaneous attack on a maneuvering target within finite time, control saturation, and control deviation.

\section{Conflict of Interests}

The authors declare that there is no conflict of interests regarding the publication of this paper.

\section{Acknowledgments}

This work was supported in part by the National Nature Science Foundation of China (nos. 61473124, 61203081 and 61174079), Doctoral Fund of Ministry of Education of China (no. 20120142120091), Fundamental Research Funds for the Central Universities of HUST (no. 2013054), and Precision Manufacturing Technology and Equipment for Metal Parts (no. 2012DFG70640).

\section{References}

[1] Z. T. Dydek, A. M. Annaswamy, and E. Lavretsky, "Adaptive configuration control of multiple UAVs," Control Engineering Practice, vol. 21, no. 8, pp. 1043-1052, 2013.

[2] W. Ren, R. W. Beard, and E. M. Atkins, "Information consensus in multivehicle cooperative control," IEEE Control Systems Magazine, vol. 27, no. 2, pp. 71-82, 2007.

[3] M. Lundell, J. Tang, T. Hogan, and K. Nygard, "Agent-oriented simulation of cooperative UAV missions," WSEAS Transactions on Systems, vol. 5, no. 4, pp. 781-786, 2006.

[4] M. Bando and A. Ichikawa, "Engineering notes active formation flying along an elliptic orbit," Journal of Guidance, Control, and Dynamics, vol. 36, no. 1, pp. 324-332, 2013.

[5] H. S. Su, G. R. Chen, X. F. Wang, and Z. L. Lin, "Adaptive secondorder consensus of networked mobile agents with nonlinear dynamics," Automatica, vol. 47, no. 2, pp. 368-375, 2011.

[6] F. Pasqualetti, D. Borra, and F. Bullo, "Consensus networks over finite fields," Automatica, vol. 50, no. 2, pp. 349-358, 2014.

[7] T. W. McLain and R. W. Beard, "Coordination variables, coordination functions, and cooperative timing missions," Journal of Guidance, Control, and Dynamics, vol. 28, no. 1, pp. 150-161, 2005.

[8] I.-S. Jeon, J.-I. Lee, and M.-J. Tahk, "Impact-time-control guidance law for anti-ship missiles," IEEE Transactions on Control Systems Technology, vol. 14, no. 2, pp. 260-266, 2006.

[9] I.-S. Jeon, J.-I. Lee, and M.-J. Tahk, "Homing guidance law for cooperative attack of multiple missiles," Journal of Guidance, Control, and Dynamics, vol. 33, no. 1, pp. 275-280, 2010.

[10] R. Olfati-Saber and R. M. Murray, "Consensus problems in networks of agents with switching topology and time-delays," IEEE Transactions on Automatic Control, vol. 49, no. 9, pp. 15201533, 2004.

[11] S. Zhao and R. Zhou, "Cooperative guidance for multimissile salvo attack," Chinese Journal of Aeronautics, vol. 21, no. 6, pp. 533-539, 2008.

[12] S. Vitaly and S. Tal, "Cooperative optimal guidance laws for imposing a relative intercept angle," in Proceedings of the AIAA Guidance, Navigation, and Control Conference, Minneapolis, Minn, USA, August 2012.

[13] E. Zhao, S. Wang, T. Chao, and M. Yang, "Multiple missiles cooperative guidance based on leader-follower strategy," in Proceedings of the 6th IEEE Chinese Guidance, Navigation and Control Conference (CGNCC '14), pp. 1163-1167, Yantai, China, August 2014.

[14] X. Wang, Y. Zheng, and H. Lin, "Integrated guidance and control law for cooperative attack of multiple missiles," Aerospace Science and Technology, vol. 42, pp. 1-11, 2015.

[15] I.-J. Ha, J.-S. Hur, M.-S. Ko, and T.-L. Song, "Performance analysis of PNG laws for randomly maneuvering targets," IEEE Transactions on Aerospace and Electronic Systems, vol. 26, no. 5, pp. 713-721, 1990.

[16] G. M. Siouris, Missile Guidance and Control System, Springer, New York, NY, USA, 2004.

[17] N. Dhananjay and D. Ghose, "Accurate time-to-go estimation for proportional navigation guidance," Journal of Guidance, Control, and Dynamics, vol. 37, no. 4, pp. 1378-1383, 2014.

[18] S. A. Murtaugh and H. E. Criel, "Fundamentals of proportional navigation," IEEE Spectrum, vol. 3, no. 12, pp. 75-85, 1966.

[19] M.-J. Tahk, C.-K. Ryoo, and H. Cho, "Recursive time-to-go estimation for homing guidance missiles," IEEE Transactions on Aerospace and Electronic Systems, vol. 38, no. 1, pp. 13-24, 2002. 
[20] L. Vincent, "Time-to-go estimate for missile guidance," in Proceedings of the AIAA Guidance, Navigation, and Control Conference and Exhibit, San Francisco, Calif, USA, August 2005.

[21] G.-B. Huang, Q.-Y. Zhu, and C.-K. Siew, "Extreme learning machine: theory and applications," Neurocomputing, vol. 70, no. 1-3, pp. 489-501, 2006.

[22] P. Zarchan, Tactical and Strategic Missile Guidance, vol. 239 of Progress in Astronautics and Aeronautics, AIAA, Reston, Va, USA, 2012.

[23] C. Godsil and G. Royle, Algebraic Graph Theory, Springer, New York, NY, USA, 2001. 

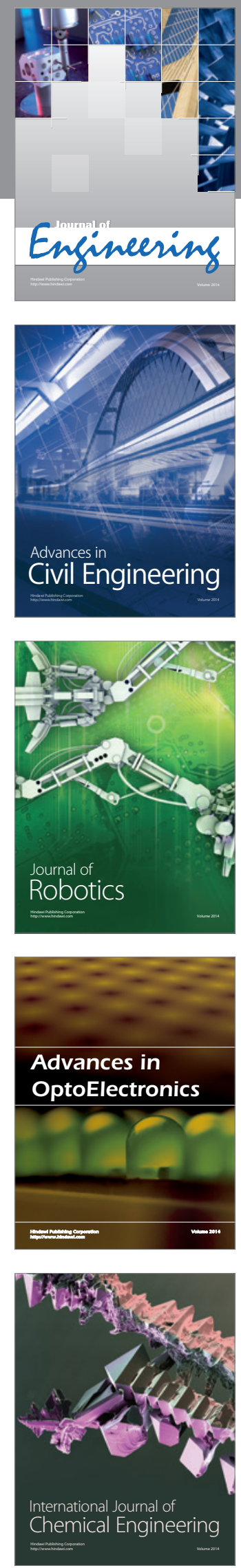

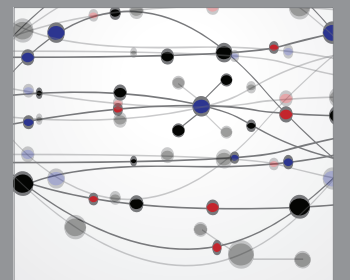

The Scientific World Journal
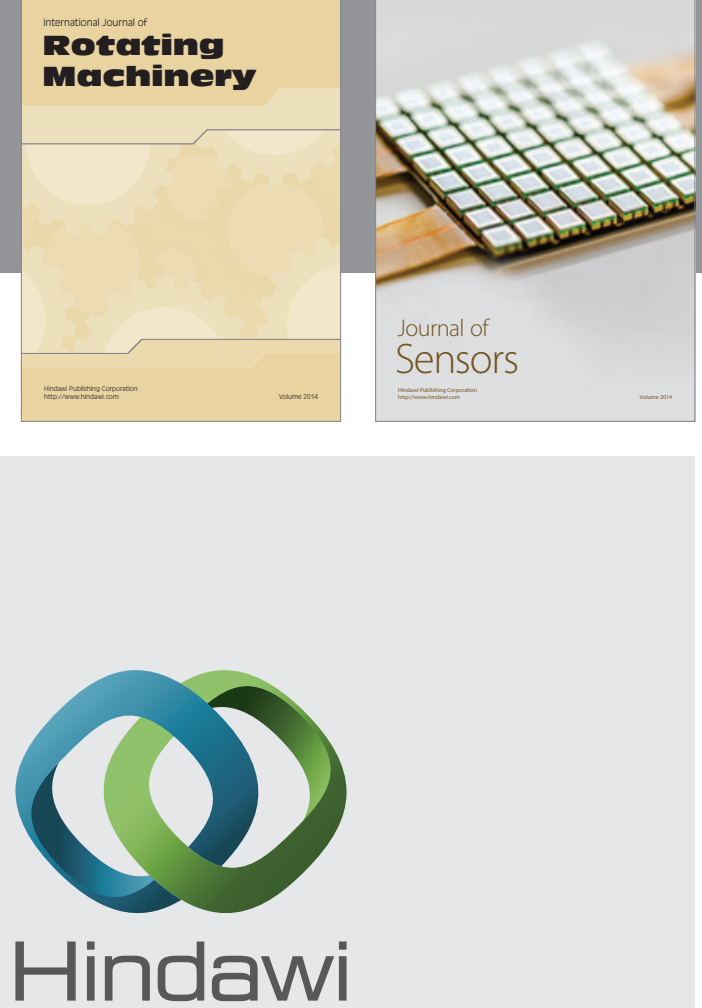

Submit your manuscripts at http://www.hindawi.com
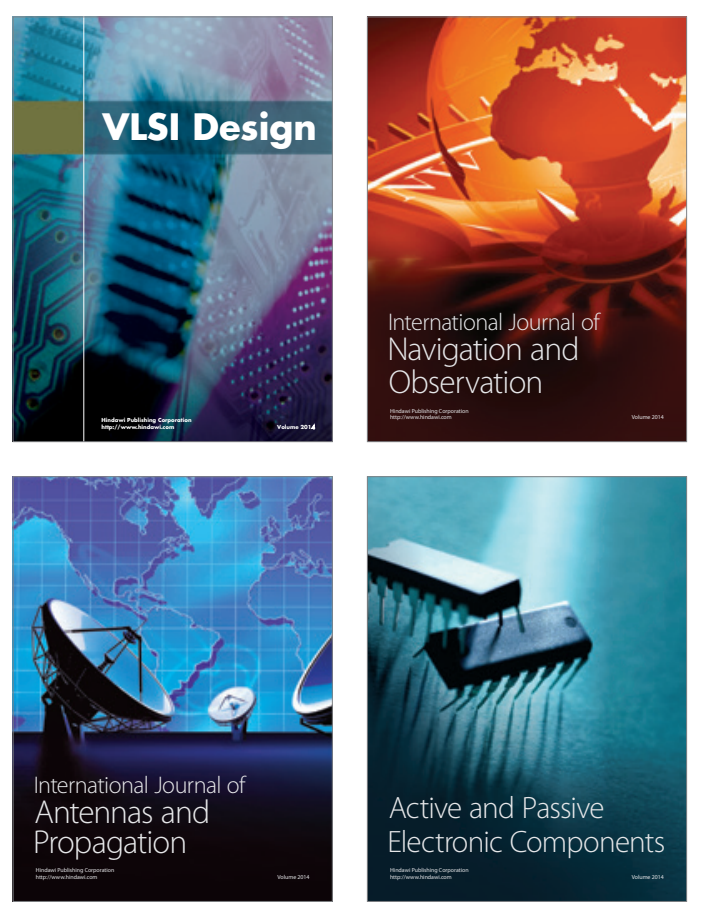
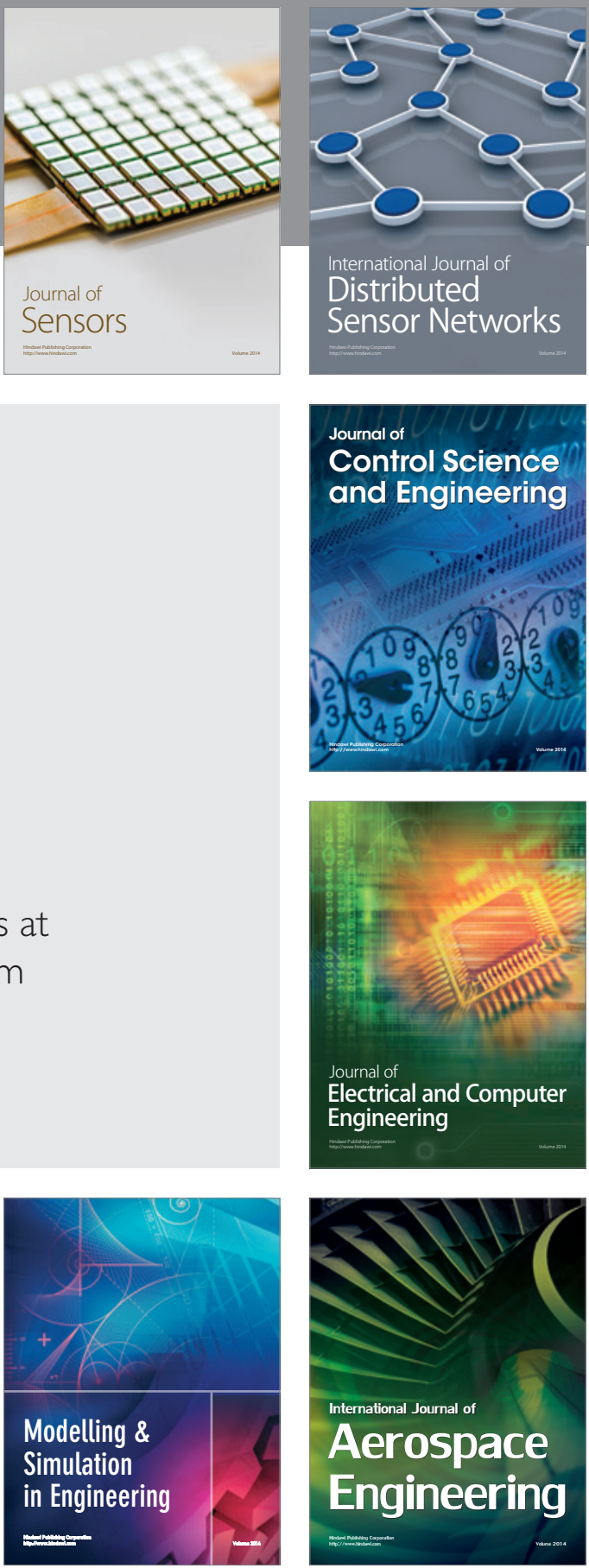

Journal of

Control Science

and Engineering
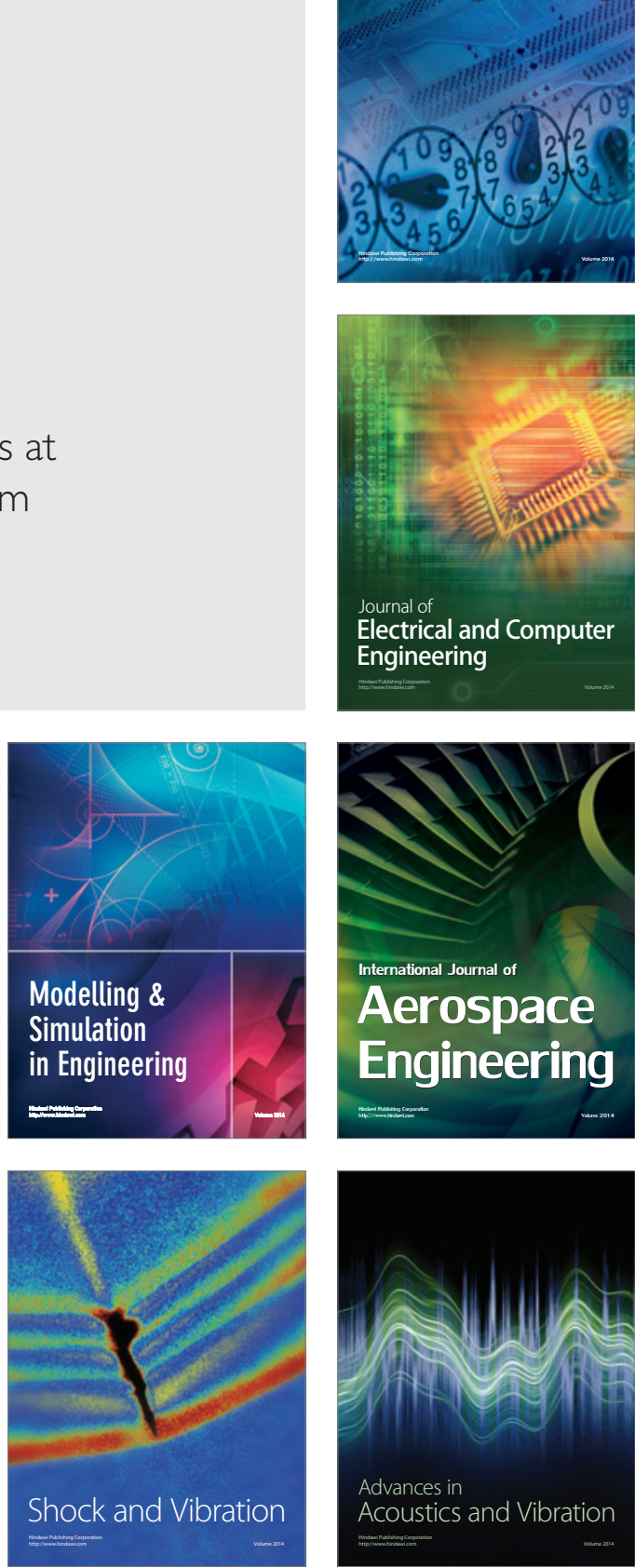